\title{
Ultraviolet Absorption Spectral Scan and Fluorescence Emission Spectral Scan Analysis: Potential Tests with Diagnostic Utility in Porphyria
}

\author{
${ }^{1}$ Archana Nimesh, ${ }^{2}$ Vibhuti Agarwal, ${ }^{3}$ Seema Garg, ${ }^{4}$ Mohit Mehndiratta
}

\begin{abstract}
Introduction: Symptoms of porphyria usually overlap with other clinical conditions, thereby leading to misdiagnosis and inappropriate treatment, especially when patient presents with uncommon features. An accurate diagnosis of porphyria can be made only when enzyme defects can be detected, which is costly and not easily available. Classically, Ehrlich's test used to screen porphyria has certain disadvantages too. Hence, we planned this study.
\end{abstract}

Aim: To develop comparatively simpler diagnostic tests feasible at tertiary care centers to work up porphyria cases.

Materials and methods: A suspected case of porphyria was screened using Ehrlich's test. Thereafter, patient's urine and serum samples were analyzed by ultraviolet (UV) absorption spectral scan and fluorescence emission spectral scan using multimode microplate reader and compared against normal controls to validate the results.

Results: The UV absorption spectral scan revealed a small peak at $410 \mathrm{~nm}$ for patient's urine sample, which intensified on acidification. The UV absorption spectral scan for patient's serum also showed absorbance peak at $405 \mathrm{~nm}$ vs normal serum. The fluorescence emission spectral scan of urine and serum of patient revealed a sharp emission peak at $626 \mathrm{~nm}$, which was not present in respective samples of control. Fluorescence emission in patient's urine sample was 10.5 times more vs normal urine.

Conclusion and clinical significance: Novel methods like UV absorption spectral scan and fluorescence emission spectral scan using patient's urine and serum samples can be developed as diagnostic tests considering their practicality and affordability. Further, an algorithm formulated based on clinical features and basic lab tests can also identify the type of porphyria.

Keywords: Diagnosis, Porphyria, Porphyria cutanea tarda, Variegate porphyria.

How to cite this article: Nimesh A, Agarwal V, Garg S, Mehndiratta M. Ultraviolet Absorption Spectral Scan and Fluorescence

${ }^{1}$ Senior Demonstrator, ${ }^{2}$ Senior Resident, ${ }^{3}$ Assistant Professor ${ }^{4}$ Associate Professor

${ }^{1-4}$ Department of Biochemistry, University College of Medical Sciences, New Delhi, India

Corresponding Author: Seema Garg, Assistant Professor Department of Biochemistry, University College of Medical Sciences, New Delhi, India, Phone: +919891365321, e-mail: seegarg@yahoo.com
Emission Spectral Scan Analysis: Potential Tests with Diagnostic Utility in Porphyria. Indian J Med Biochem 2017;21(2):136-141.

Source of support: Nil

Conflict of interest: None

\section{INTRODUCTION}

Porphyria is a clinical condition that occurs due to a defect in heme synthesis. This condition may be genetic or acquired in nature. ${ }^{1}$ The signs and symptoms of patients suffering from porphyria usually overlap with other clinical conditions, thereby leading to misdiagnosis and inappropriate treatment in most patients. The diagnosis becomes even more challenging when a case of porphyria presents with an unusual presentation. Thus, most of the cases remain underreported due to misdiagnosis. Hence, the true prevalence of porphyria is not known. The literature search revealed that incidence of porphyria cutanea tarda is 40 new cases per 1 million people per year. ${ }^{2}$ The prevalence of acute intermittent porphyria in the general adult population ranges between 1 and 8 per lakh., An accurate and definite diagnosis of porphyria and its types can be made only when enzyme defect or mutation can be detected. However, the availability and cost of these tests is an important practical problem encountered in a country like India. In this article, we discuss a probable case of porphyria cutanea tarda/variegate porphyria based upon the clinical picture of patient. These cases can be easily confused with other skin diseases due to their presentation in the form of photosensitive lesions. Hence, we wish to propose a simpler diagnostic approach based on easily available facilities to diagnose patients with porphyria.

\section{CASE REPORT}

A 22-year-old male patient, carpenter by occupation, reported to the dermatology department with certain skin lesions on photo-exposed parts of the body. A differential diagnosis of epidermolysis bullosa dystrophica, epidermolysis bullosa acquisita, and pleomorphic light eruption was made. The patient was investigated using laboratory tests. The investigation reports did not correlate with the clinical picture. Therefore, the patient was made to undergo several further investigations. Eventually, a 
probability of porphyria was suspected by the dermatologist, and the patient was referred to the Department of Biochemistry for diagnostic workup for the same.

The patient was evaluated by us based upon history, clinical examination, and laboratory tests (Tables 1 and 2) he had undergone. The patient presented with chief complaints of skin lesions on face, dorsum of hands, and feet for last 6 months, which appeared and worsened on exposure to sunlight. Patient described the lesions as fluid-filled eruptions that would rupture spontaneously leaving behind sore skin and patches of pigmentation. He also has a chronic history of alcohol intake for last 10 years. He had been drinking a local variety of alcoholcontaining drink (around 1 L per day). He described in his history an interesting feature that his lesions improve on abstaining from alcohol. At the time of presenting to the

Table 1: Blood and urine investigations of patient

\begin{tabular}{|c|c|c|}
\hline Blood investigations & Result & $\overline{\text { Interpretation }}$ \\
\hline Hemoglobin & $13.6 \mathrm{gm} \%$ & Normal \\
\hline Hematocrit & $43.6 \%$ & Normal \\
\hline RBC count & 5.1 million cells/cumm & Normal \\
\hline MCV & $84 \mathrm{fL}$ & Normal \\
\hline $\mathrm{MCH}$ & $26.4 \mathrm{pg}$ & Normal \\
\hline $\mathrm{MCHC}$ & $31.5 \mathrm{~g} / \mathrm{dL}$ & Normal \\
\hline DLC & $\begin{array}{l}\text { Neutrophils } 65 \% \text {, } \\
\text { lymphocytes } 25 \% \text {, } \\
\text { monocytes } 7 \% \text {, } \\
\text { eosinophils } 3 \%\end{array}$ & Normal \\
\hline TLC & 4800/cumm & Normal \\
\hline Platelet count & 1.78 lakh/cumm & Normal \\
\hline ESR & 106 & \\
\hline Peripheral smear picture & $\begin{array}{l}\text { Normocytic } \\
\text { normochromic }\end{array}$ & \\
\hline Hepatitis B antigen (ELISA) & Nonreactive & Normal \\
\hline HCV antibody (ELISA) & Nonreactive & Normal \\
\hline Serum bilirubin (total) & $0.6 \mathrm{mg} / \mathrm{dL}$ & Normal \\
\hline Serum bilirubin (direct) & $0.1 \mathrm{mg} / \mathrm{dL}$ & Normal \\
\hline ALP & $74 \mathrm{IU} / \mathrm{L}$ & Normal \\
\hline AST & $36 \mathrm{IU} / \mathrm{L}$ & Normal \\
\hline ALT & $46 \mathrm{IU} / \mathrm{L}$ & Normal \\
\hline Total serum protein & $7.3 \mathrm{~g} / \mathrm{dL}$ & Normal \\
\hline Serum albumin & $3.9 \mathrm{~g} / \mathrm{dL}$ & Normal \\
\hline \multicolumn{3}{|l|}{ Urine investigations } \\
\hline Color & Straw colored & Normal \\
\hline Appearance & Transparent & Normal \\
\hline Odor & Ammonical & Normal \\
\hline Specific gravity & 1.020 & Normal \\
\hline $\begin{array}{l}\text { Sugar, albumin, ketone } \\
\text { bodies, bile salts and bile } \\
\text { pigments }\end{array}$ & Nil & Normal \\
\hline $\begin{array}{l}\text { Pus cells, RBCs, cysts, } \\
\text { hemoglobin }\end{array}$ & Nil & Normal \\
\hline
\end{tabular}

RBC: Red blood cell; MCV: Mean corpuscular volume; $\mathrm{MCH}$ : Mean corpuscular hemoglobin; MCHC: Mean corpuscular hemoglobin concentration; DLC: Differential leukocyte count; TLC: Total leukocyte count; ESR: Erythrocyte sedimentation rate; HCV: Hepatitis C virus; ALP: Alkaline phosphatase; AST: Aspartate transaminase; ALT: Alanine transaminase
Table 2: Special investigations of patient

A: Direct immunofluorescence report of skin epidermis

$\begin{array}{lll}1 & \lg G & 2+ \\ 2 & \lg \mathrm{A} & \text { Negative } \\ 3 & \lg \mathrm{M} & \text { Negative } \\ 4 & \mathrm{C} 3 & \text { Negative } \\ 5 & \mathrm{~F} & \text { Negative }\end{array}$

B. Skin biopsy: Skin shows hyperkeratosis, mild acanthosis with upper dermal sclerosis, and mild perivascular inflammation

C. Onychoscopy (photo-onycholysis): Homogeneous or striated onycholytic bands with proximal erythema in most of the nails. Some nails showed alternating transverse erythema and leukonychia bands

D. Iron profile study: Serum iron $91 \mu \mathrm{g} / \mathrm{dL}$, TIBC $454 \mu \mathrm{g} / \mathrm{dL}, \%$ saturation $20.5 \%$ (Interpretation: Iron profile normal)

E. Ultrasound (USG) abdomen/pelvis: Normal

F. Ehrlich's test for urine: Positive

IgG: Immunoglobulin G; IgA: Immunoglobulin A; IgM: Immunoglobulin M; C3: Complement 3; F: Fibrinogen; TIBC: Rotal iron binding capacity

hospital, he had been abstaining from alcohol for the last 3 months. His lesions also improved on topical application of a sunscreen prescribed to him by the dermatologist. He does not have any history of neuropsychiatric symptoms or abdominal pain. There is no family history of such lesions as well.

On further investigations, he described that around 3 years back, he had developed severe chest pain for which he was admitted in the emergency ward of a nearby hospital. There he had undergone some investigations like chest X-ray, electrocardiogram, and blood tests following which he was given some treatment at the hospital that had flared up a similar episode of skin lesion on his photo-exposed parts. The patient got apprehensive and discontinued the treatment within a week. The lesions had lasted for around 3 months then, and healed gradually. The patient remained asymptomatic thereafter, until the recent episode.

On physical examination, the patient revealed that lesions were of erosive and ulcerative type with erythematous base (Fig. 1A). Size of lesions ranged from $2 \mathrm{~mm}$ to $3 \mathrm{~cm}$. The lesions were found to be present on face and dorsum of hands (Figs 1A and B respectively). The skin also showed patches of postinflammatory hypoand hyperpigmented areas (Fig. 1B). The healed lesions were characterized by crusting and scarring (Fig. 1B). Systemic clinical examination revealed no abnormality.

The history and clinical examination is, thus, suggestive of a sporadic case of acquired porphyria with alcohol and drugs being the precipitating factors. ${ }^{1}$ However, the past episode suggestive of constrictive pericarditis, an uncommon presentation of porphyria cutanea tarda cannot be ascertained due to nonavailability of previous medical records. ${ }^{5}$ Thus, from the above discussion, it is 

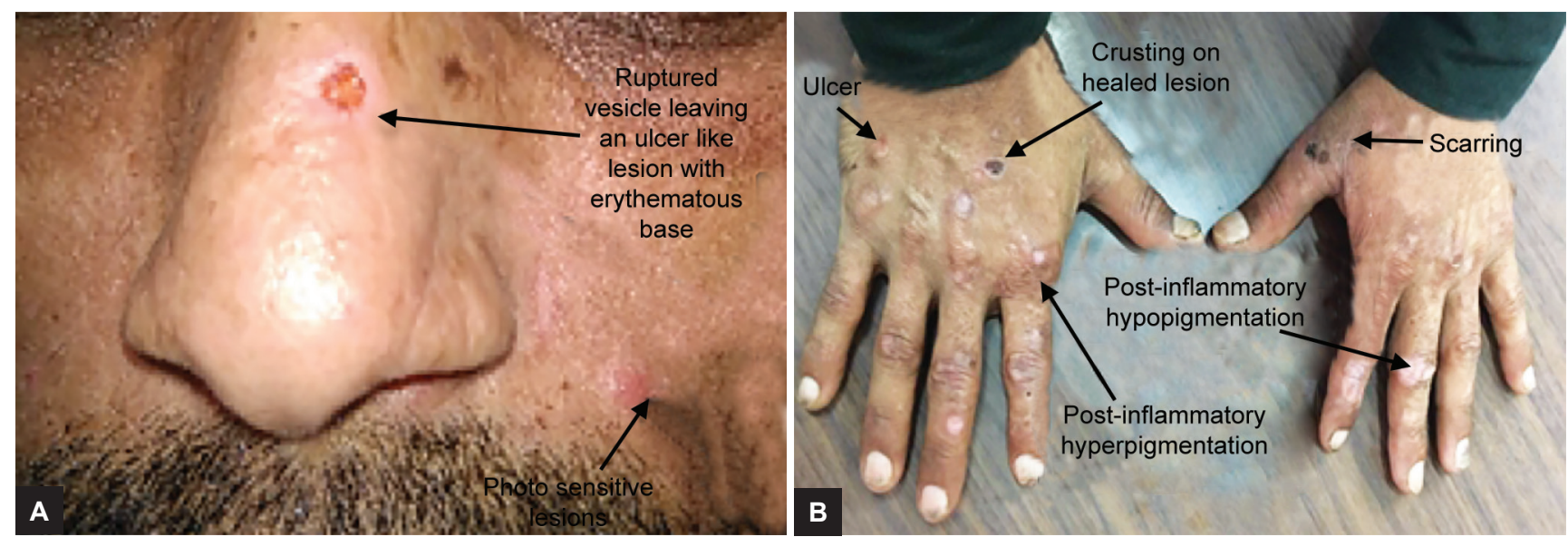

Figs $1 \mathrm{~A}$ and B: Skin lesions in a case of porphyria: (A) Blisters and erosions on face; and (B) skin lesions on dorsa of hands

evident that a suspected case of porphyria is usually and unnecessarily subjected to a wide number of tests (Tables 1 and 2), which could be financially burdening to the patient and, in most instances, porphyria still remains the diagnosis by exclusion.

From literature it is known that suspected cases of porphyria can undergo a basic screening test called Ehrlich's test as discussed here. The method to carry out Ehrlich's tests is described in Varley's Practical Clinical Biochemistry. ${ }^{6}$ Development of rose pink color in aqueous layer is indicative of presence of porphobilinogen (PBG). However, development of rose pink color in the chloroform layer occurs due to urobilinogen also, but is seen only when the amount of urobilinogen in the urine sample is very high as seen in hemolytic anemias. In our study, patient's urine sample tested positive for Ehrlich's test (aqueous layer turned rose pink, thereby, indicating presence of PBG) giving a clue that the patient could be a case of porphyria. But, this test has its own disadvantages. A negative Ehrlich's test does not rule out the possibility of porphyria totally. Ehrlich's test is answered positive in a case of porphyria only when the concentration of PBG exceeds 10 to 20 times more than the normal. ${ }^{7}$ Moreover, Ehrlich's test requires certain reagents for the urine testing like Ehrlich's reagent (containing $p$-amino benzaldehyde), saturated solution of sodium acetate, chloroform, and freshly voided urine samples of the suspected patient and healthy control. Secondly, these reagents have to be prepared fresh. It being a manual method, takes comparatively longer time to process the samples. Though the possibility and feasibility of carrying out Ehrlich's test may be present in medical colleges where reagents may be available from an academic point of view, most of the diagnostic centers do not have these reagents to carry out the test.

Another method to screen a case of porphyria can be by using Watson-Schwartz method. This method is being followed in the United States, but it has a major limitation of poor sensitivity. ${ }^{8}$ Hoesch test is also recommended for screening cases of porphyria in some countries. However, this test shows false-positive results. Before the diagnosis of "inducible" porphyria is made, a positive Hoesch test requires that indoles, indoleacetic acid, methyldopa, endstage alcoholic malnutrition, and phenazopyridine $\mathrm{HCl}$ be excluded, to avoid misinterpretation. ${ }^{9}$ Both WatsonSchwartz method and Hoesch tests are thus considered complicated methods to test for PBGs. ${ }^{9}$

Considering the drawbacks of these available methods, there is a need to have a simpler diagnostic approach for working up cases of porphyria, especially in stand-alone laboratories. Hence, it prompted us to carry out this study to find out alternate methods for diagnosing patients of porphyria.

\section{MATERIALS AND METHODS}

This work was carried out in the Department of Biochemistry Guru Teg Bahadur Hospital and University College of Medical Sciences, Delhi after taking written informed consent from the patient. Porphyrinogens have the property to absorb 400 to $410 \mathrm{~nm}$ wavelength and emit out 619,626 , or $634 \mathrm{~nm}$ wavelengths. ${ }^{10}$ This property has been exploited by us to discover two methods, namely UV absorption spectral scan analysis and fluorescence emission spectral scan analysis. The procedure to carry out these tests and the interpretation of the results so generated are being described here.

An instrument called multimode microplate reader (based upon the principle of spectrophotometer) and a microtiter plate are required to perform these tests. The samples to be analyzed ( $200 \mu \mathrm{L}$ was taken) were filled in the wells of the microtiter plate, and readings for absorbance and fluorescence emissions were recorded in the multimode microplate reader (synergy H1 hybrid multimode reader by Biotek company, USA) at a series of wavelengths with an increment of $1 \mathrm{~nm}$ (spectral scan). A transparent 
microtiter plate (by Tarson, USA) for measuring absorbance and a black microtiter (by Tarson, USA) plate for measuring fluorescence emission were used.

\section{Ultraviolet Absorption Spectral Scan Analysis}

The absorption scan was carried out at a series of wavelengths (ranging from 350 to $500 \mathrm{~nm}$ ) in the multimode microplate reader for urine and serum samples of the patient as well as normal individual (which served as control) and the graphs were recorded. Distilled water served as blank. The above experiment was repeated after acidifying the patient's urine sample and normal urine sample by using $5 \%$ hydrochloric acid $(1: 1 \mathrm{v} / \mathrm{v}$ ratio of urine and $5 \% \mathrm{HCl}$ was used) and graph was recorded.

\section{Fluorescence Emission Spectral Scan Analysis}

Patient's urine sample, normal individual's urine sample (control), and distilled water (blank) were excited at $410 \mathrm{~nm}$ and emission values at 619,626 , and $634 \mathrm{~nm}$ were recorded. From literature, it is known that porphyrinogens emit these wavelengths upon excitation with $410 \mathrm{~nm} .{ }^{10}$ The urine samples of patient and control were further analyzed

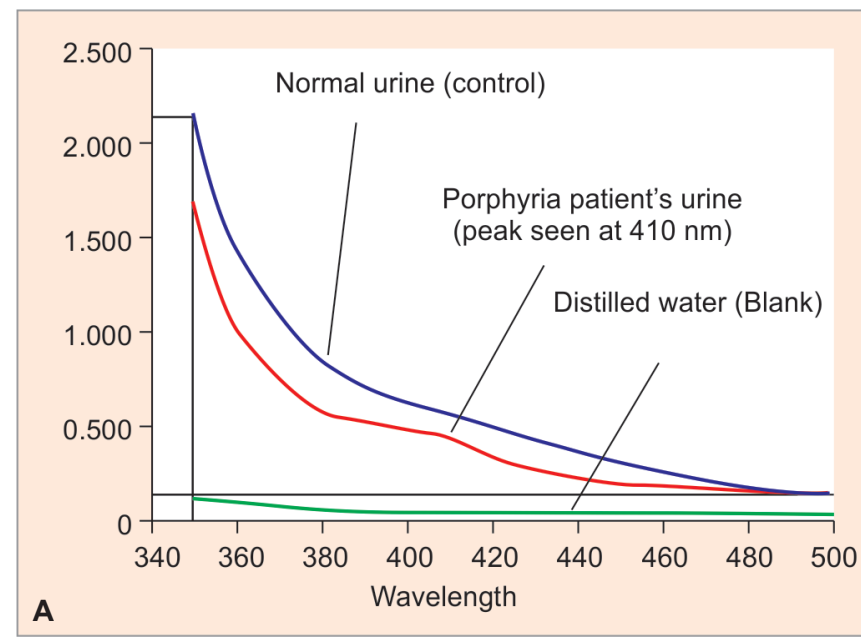

for spectral scan of the fluorescence emission at a series of wavelengths (ranging from 450 to $700 \mathrm{~nm}$ ), and the findings were recorded as graph. The fluorescence emission scan experiment was repeated again by using the serum samples of the patient and two normal subjects (serving as controls) this time, and the findings were recorded as graph.

\section{RESULTS}

The graph shown in Graph 1A after UV absorption spectral scan revealed a slight peak at $410 \mathrm{~nm}$ for the patient's urine sample, whereas no such peak was observed for control sample (normal urine sample). Graph 1B obtained for UV absorption spectral scan after the acidification of patient's urine sample and normal individual's urine sample showed that the peak was intensified at $405 \mathrm{~nm}$. The UV absorption spectral scan for serum samples also showed that the absorbance peaked at around $405 \mathrm{~nm}$ for the patient's serum sample only (Graph 1C).

The results of fluorescence emission measurement (upon excitation with $410 \mathrm{~nm}$ ) recorded at 619, 626, and $634 \mathrm{~nm}$ showed that maximum fluorescence emission value (796.80) for patient's urine sample was found to

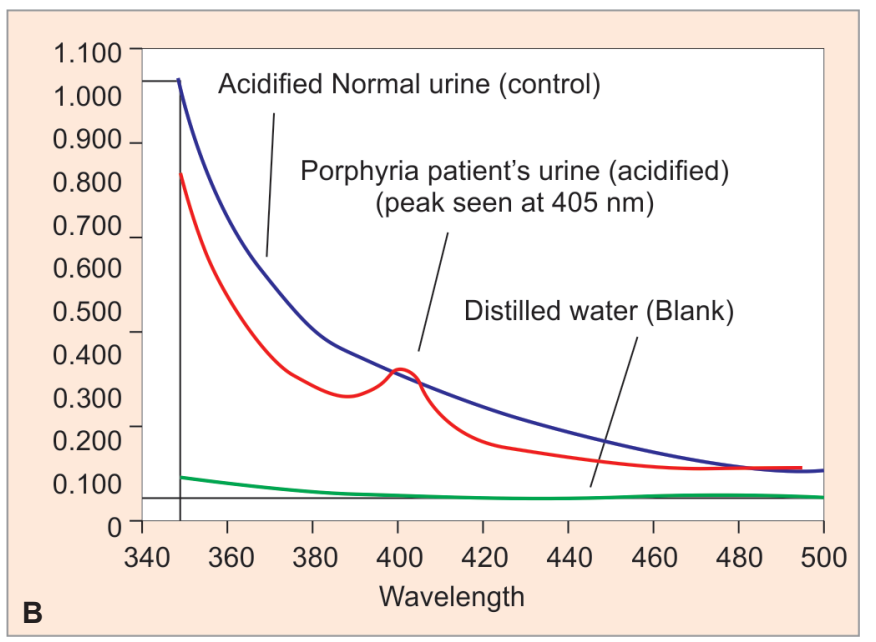

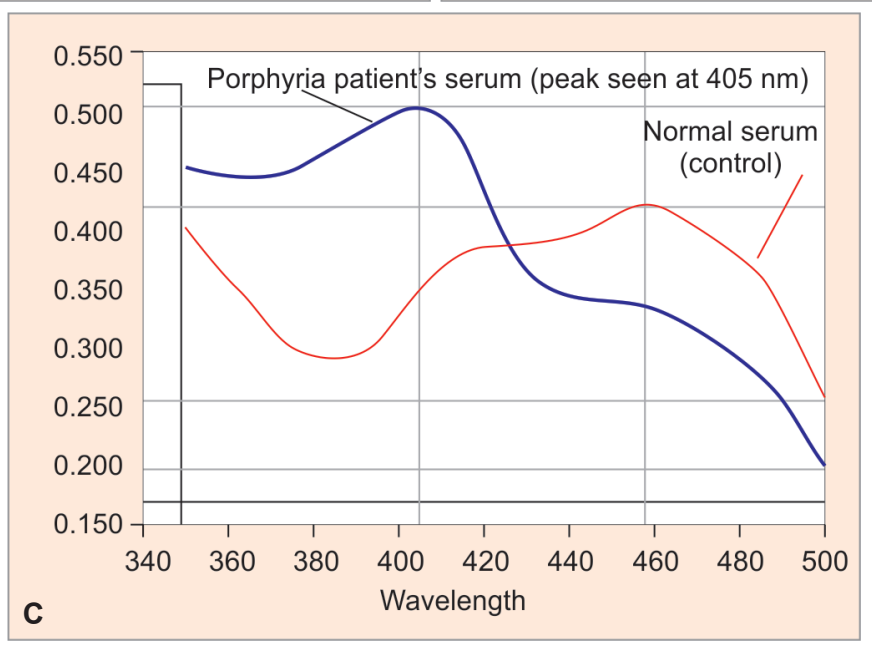

Graphs 1A to C: Absorption spectra using: (A) Unacidified urine sample; (B) acidified urine sample; and (C) serum sample of a case of porphyria in comparison to control and blank. The wavelengths scanned for absorption spectrum were 350 to $500 \mathrm{~nm}$ with $1 \mathrm{~nm}$ increment 


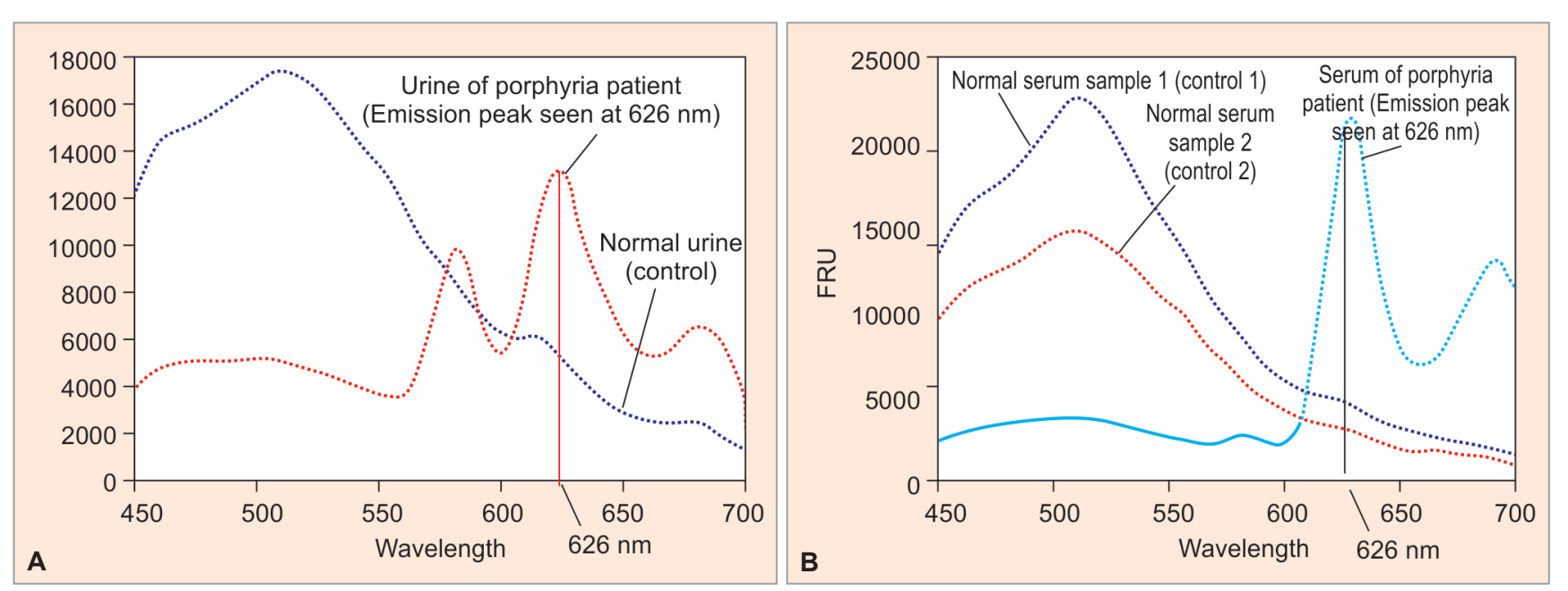

Graphs 2A and B: Fluorescence emission spectra of: (A)Acidified urine sample; and (B) serum sample of a case of porphyria in comparison to control samples. ${ }^{*}$ The wavelength series scanned for fluorescence emission spectrum were from 450 to $700 \mathrm{~nm}$ with $1 \mathrm{~nm}$ increment

be at $626 \mathrm{~nm}$ vs normal urine (75.42) and distilled water (0.48). This fluorescence emission value at $626 \mathrm{~nm}$ for patient's urine was found to be 10.5 times more than that of normal urine sample. The fluorescence emission spectral scan using the urine samples revealed a sharp emission peak at $626 \mathrm{~nm}$ (Graph 2A) for the patient's urine vs control. The fluorescence emission spectrum analysis of patient's serum sample $v$ s two normal serum samples (controls) also revealed a sharp emission peak at $626 \mathrm{~nm}$ for the patient's serum sample only (Graph 2B).

\section{DISCUSSION}

We were able to confirm the diagnosis of porphyria in our patient using UV absorption spectral scan and fluorescence emission spectral scan. From the above experiments, it is clear that examining an acidified urine sample of the suspected patient and comparing it with an acidified normal urine sample using UV absorption spectral scan and fluorescence emission spectral scan can be easily done without any requirement for special reagents. These tests on urine samples are absolutely noninvasive, cheaper, and are practically feasible for any stand-alone diagnostic lab or a tertiary care center to perform. Alternately, the same tests can also be carried out using patient's serum sample and comparing it with normal serum sample. These UV absorption and fluorescence emission spectra studies require a multimode microplate reader and a simple microtiter plate, which are very commonly available at most of the diagnostic centers. Repeating these tests is not cumbersome unlike Ehrlich's test and other available tests. Moreover, the sample is not consumed at all in spectral scan and can be reused if scan is to be repeated. The turn-around time for any lab to generate these results using urine samples shall barely exceed 20 minutes. Thus, we propose that $\mathrm{UV}$ absorption and fluorescence emission spectrum studies on urine samples can be developed as diagnostic tests in future in the stand-alone laboratories considering the practicality and affordability of these tests vs the studies to detect genetic mutations and enzyme assays in porphyria patients.

By using UV absorption and fluorescence emission spectrum studies, though we can promptly diagnose cases of porphyria, if it is further desired to know the type of porphyria the patient has, then an algorithm formulated by us (Flow Chart 1) based on clinical features and basic lab tests can be used. Differential diagnoses for porphyria cutanea tarda/variegate porphyria include other blistering disorders, such as polymorphic light eruptions, bullous lupus erythematosus, epidermolysis bullosa acquisita, photo-induced bullous drug reactions, and solar urticaria. All of these conditions have normal (i.e., negative) urine, fecal, and serum porphyrin levels. ${ }^{11}$ These dermatological conditions can be worked up accordingly if UV absorption and fluorescence emission spectrum studies are found to be negative.

A limitation of our study is that porphyria being a rare genetic disease, we could not enroll more patients in our research.

\section{CONCLUSION}

We have described a case of porphyria in our article whose prevalence is very rare. This case is probably of the acquired type, who has developed porphyria secondary to chronic alcohol intake (ethanol, a porphyria causing agent). We have also found that UV absorption spectral scan and fluorescence emission spectral scan have the potential to be developed as diagnostic tests in future, considering the practicality and affordability of these tests vs the gold standard studies to detect genetic mutations and enzymatic assays in porphyria patients. 
Flow Chart 1: Algorithm to aid in identifying type of porphyria based on clinical symptoms and lab tests. ${ }^{*}$ ALA synthase and ALA dehydratase deficiency do not show emission peak; ${ }^{* *}$ Enzymatic assays or molecular diagnostics will be confirmatory for diagnosis; ***UV: Ultraviolet; USG: Ultrasonography; ALA: Aminolevulinic acid

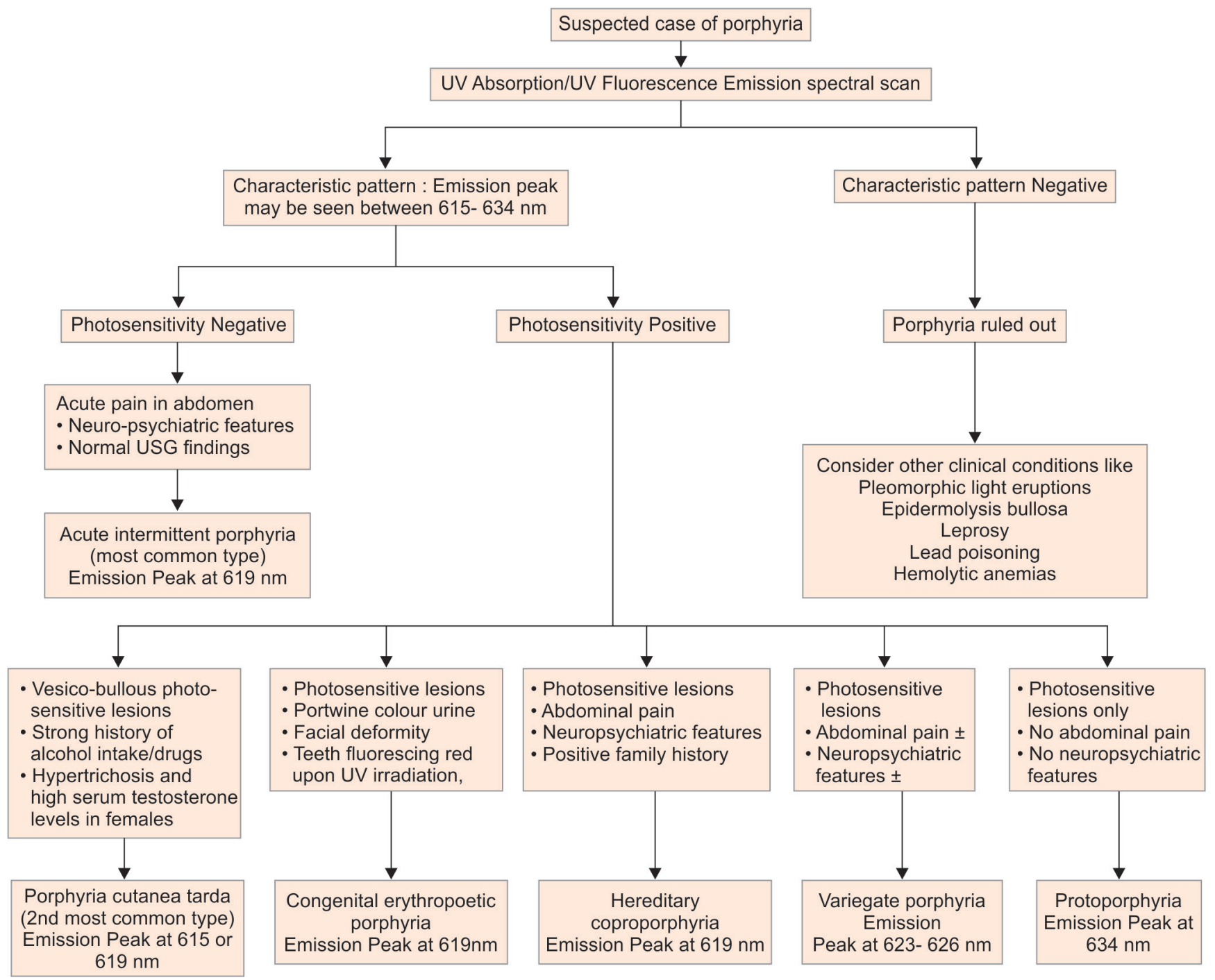

\section{CLINICAL SIGNIFICANCE}

The UV absorption spectral scan and fluorescence emission spectral scan are easy, affordable, and feasible techniques that can be performed in tertiary care centers or diagnostic centers that can help in prompt and definite diagnosis of porphyria.

\section{ACKNOWLEDGMENT}

Authors would like to thank their patients for participating in the research work.

\section{REFERENCES}

1. Goerz G, Merk H. Porphyria cutanea tarda (PCT). Z Hautkr 1985 Jan;60(1-2):137-146.

2. Merk HF. Porphyria cutanea tarda. Hautarzt 2016 Mar;67(3): 207-210.

3. Ackner B, Cooper JE, Gray CH, Kelly M. Acute porphyria: a neuropsychiatric and biochemical study. J Psychosom Res 1962 Jan-Mar;6(1):1-24.
4. Goldberg A. Acute intermittent porphyria: a study of 50 cases. Q J Med 1959 Apr;28(110):183-209.

5. Jasleen K, Sharma N, Kalsy J, Sharma M. Porphyria cutanea tarda with constrictive pericarditis: a rare association. Case Rep Dermatol Med 2012 Jan;2012:972162.

6. Varley, H.; Gowenlock, AH.; Bell, M. Practical clinical biochemistry. Vol. 1. 5th ed. London: Heinemann; 1980. p. 958.

7. Schreiber WE, Jamani A, Pudek MR. Screening tests for porphobilinogen are insensitive. The problem and its solution. Am J Clin Pathol 1989 Nov;92(5):644-649.

8. Buttery JE, Carrera AM, Pannall PR. Reliability of the porphobilinogen screening assay. Pathology 1990 Oct;22(4): 197-198.

9. Pierach CA, Cardinal R, Bossenmaier I, Watson CJ. Comparison of the Hoesch and the Watson-Schwartz tests for urinary porphobilinogen. Clin Chem 1977 Sep;23(9):1666-1668.

10. Walsh DS, Beard JS, James WD. Fluorescent spectrophotometric analysis in the evaluation of porphyria. JAMA 1994 Nov 23;272(20):1580-1581.

11. Ting P, Adams S. Dermacase. Can you identify this condition? Porphyria cutanea tarda. Can Fam Physician 2013 Jul;59(7):749, 753-755. 